\title{
Developing an Easy-to-Use Simulator to Thermodynamic Design of Gas Condensate Reservoir's Separators
}

\author{
Ahmadreza Ejraei Bakyani ${ }^{*}$, Samira Heidari², Alireza Rasti ${ }^{3}$, Azadeh Namdarpoor1 \\ ${ }^{1}$ Department of Petroleum Engineering, School of Chemical, Petroleum, and Gas Engineering, Shiraz University, Shiraz, Iran \\ ${ }^{2}$ Department of Chemical Engineering, School of Chemical, Petroleum, and Gas Engineering, Shiraz University, Shiraz, Iran \\ ${ }^{3}$ Petropars Operation \& Management Company, Shiraz, Iran \\ Email: *ahmadrezaejraei@gmail.com
}

How to cite this paper: Ejraei Bakyani, A., Heidari, S., Rasti, A. and Namdarpoor, A. (2018) Developing an Easy-to-Use Simulator to Thermodynamic Design of Gas Condensate Reservoir's Separators. Modeling and Numerical Simulation of Material Science, 8, 1-19.

https://doi.org/10.4236/mnsms.2018.81001

Received: December 26, 2017

Accepted: January 28, 2018

Published: January 31, 2018

Copyright (๑) 2018 by authors and Scientific Research Publishing Inc. This work is licensed under the Creative Commons Attribution International License (CC BY 4.0).

http://creativecommons.org/licenses/by/4.0/

(c) (i) Open Access

\begin{abstract}
Separator design in petroleum engineering is so important because of its important role in the evaluation of optimum parameters and also to achieve to maximum stock tank liquid. However, no simulator exists that simultaneously and directly optimizes the parameters "pressure", "temperature", and so on. On the other hands, Commercial simulators fix one parameter and vary another parameter to achieve the optimum conditions. So, they need long-time simulation. Moreover, gas condensate reservoirs, like another reservoirs, have this problem as well. In present paper, a self-developed simulator applied in the optimized design of gas condensate reservoir's separators by determining optimized pressure, temperature, and number of separators in order to obtain maximized tank liquid volume and minimized tank liquid density utilizing Matlab software and other commercial simulators such as Aspen-Plus, Aspen-Hysys, and PVTi to do a comparison. Also, each software was separately tested with one, two, and three separators to obtain the optimum number of separators. Additionally, Peng-Robinson equation of state (PR EOS) has been applied in the simulation. For simulation input, a set of field data of gas condensate reservoir has been utilized, as well. The results show a good compatibility of this simulator with other simulators but in so little runtime (this simulator calculates the optimum pressure and temperature in a wide range of pressures and temperatures with the help of a simultaneous optimization algorithm in one stage) and the highest stock tank liquid is calculated with this simulator in comparison to other simulators. Also, with the help of this simulator, we are able to obtain the optimum pressure, temperature, and the number of separators in the gas condensate reservoir's separators with any desired properties. Finally, this simulator optimizes the temperatures for each separator and obtains very good results despite the other simulators that fix temperatures for all separators in most times.
\end{abstract}




\section{Keywords}

Separator Design, Matlab Software, Simultaneous Algorithm, Optimum Condition, Gas Condensate Reservoir

\section{Introduction}

Gas condensate reservoirs mostly produce gas, with some liquid dropout, frequently occurring in the wellhead separators. The phase diagram shows the retrograde gas must have a temperature higher than the critical temperature. Also, the phase diagram shows the phase changes in the reservoir, while the curve line shows these changes as the fluid cools going up the wellbore and into the separator. In both cases, liquids drop out as the pressure drops below dew point pressure [1] [2].

Modeling for optimization of the conditions (pressure, temperature, and number of separators) of separators in multistage separators causes to reduce the amount of gas produced with condensate to a minimum [1]. In gas condensate reservoirs, large amounts of condensate and gas will produce in wellhead that we like to reduce amounts of gas and to obtain optimum conditions. In wellbore fluids or gas reservoirs, we face a high range of compositions that the quantity and characteristic of all of them are not known to us. Therefore, the optimized conditions of separators have to be specified by a combination of laboratory or field data and modeling. By leaving the gas phase from the liquid phases, the separator and stock tank gases have a minimum quantity. The pressure and temperature of this minimum point are referred to as the optimized pressure and temperature of the separator [2].

The separator will be modeled with the help of phase equilibrium calculations. In phase equilibrium calculation, a thermodynamic model and an optimization algorithm must be chosen. A thermodynamic model gives the relation between pressure, molar volume, and temperature for pure components and mixtures. The thermodynamic model is usually nonlinear and nonconvex and therefore, an optimization method must be utilized to find phase equilibrium [3] [4]. The method proposed by Adewumi for solving isothermal flash calculations was recommended as an optimized solving algorithm [5]. Initially, a converging sequence of upper and lower bound on the global minimum through the convex relaxation of the original problem was proposed [6]. However, the deterministic global optimization algorithm $\alpha$-based branch was applied in the fluid phase equilibrium problems as well as bound to find chemical equilibrium [7]. On another hand, an enhanced simulated annealing algorithm was proposed to verify phase stability analysis and obtain the true solution of the phase equilibrium problems in multi-component systems at high pressures [8]. But, two direct and indirect algorithms solve the phase equilibrium problem to increase the flexibility of solving algorithm in the fluid phase equilibrium systems [9]. Also, a global 
optimization method called Tunneling was suggested that is able to escape from local minima and saddle points, and it's a suitable method for many problems associated with the mathematical issues such as local minimum or/and saddle points [10].

As a result of the optimization technique, the optimization techniques were applied to directly minimize the fluid properties for a specified number of phases [11]. In accordance with the runtime issue of solving algorithms in the fluid phase equilibrium problems, a method was proposed to accelerate convergence rate of Successive substitution algorithm [10]. After that, a two-phase field flow inside an oil-gas separator with software Fluent was simulated. Based on the analysis of the two-phase flow, the authors realized the centrifugal force and the collision plays an important role in the oil-gas separation. The numerical model and the correspondent analysis are proved to be effective in the engineering design of oil-gas separators. The oil carry-over rate is greatly reduced in the modified separator [12]. Then, a new packing and newly designed Crude oil-water separator related to the physical properties of ASP products in Daqing Oilfield was proposed [13]. The orthogonal test is utilized to optimize the design of the new separator included the structure and material of coalescent packing and the new type separation efficiency of higher than $98 \%$. However, a method for optimizing separator pressures in multistage crude oil production was proposed with the help of equation of states [14]. Also, an approach for the minimization of the Gibbs free energy was developed using the linear programming that guarantees to find the global optimum within some level of precision, for any kind of thermodynamic model [15]. Additionally, a criterion for phase equilibrium is defined as: 1) the temperature and pressure of the phases are equal, 2) the chemical potentials of each component in each phase are equal, and 3) the global Gibbs free energy is a minimum [16]. As a result of novel algorithms that are able to describe the multi-phase and multi-component chemical systems such as oil-gas system either in the dissolution or the separation processes, a new model based on adaptive neuro-fuzzy inference systems (ANFIS) is developed for accurate prediction of carbon dioxide gas diffusivity in oil at elevated temperature and pressures. Also, particle swarm optimization (PSO) technique based on the stochastic search algorithms was applied to obtain the optimal ANFIS model parameters as well [17].

\section{Methodology}

As mentioned in the previous section, because of the importance of the wellhead separators as well as their parameters optimization due to some problems associated with available commercial simulators, including high cost and time consuming, as well as the lack of a simulator which particularly studies the phase behavior of fluids in gas condensate reservoirs, a new simulator is developed as below.

In this part, we develop a Matlab code to obtain the required optimum para- 
meters with the help of the followed flowchart as Figure 1 and Figure 2.

We applied some simulators to optimize the required parameters as mentioned previously to show the ability of these to optimize the separator parameters in gas condensate reservoirs and also to the comparison of these with the developed easy-to-use the simulator to show the ability of this simulator in decreasing time and cost. The existence of an algorithm that simultaneously applies to calculate the temperature and the pressure and gives an optimum temperature and pressure without manual working causes time decreasing. However, existence an algorithm that leads to higher stock tank liquid causes income increasing or cost decreasing especially in a high amount of produced liquid in surface facilities.

With each simulator, the optimum parameters were obtained and important parameters of separators fluids such as liquid and gas density, liquid and gas flow, liquid and gas enthalpy, liquid and gas entropy, and average molecular

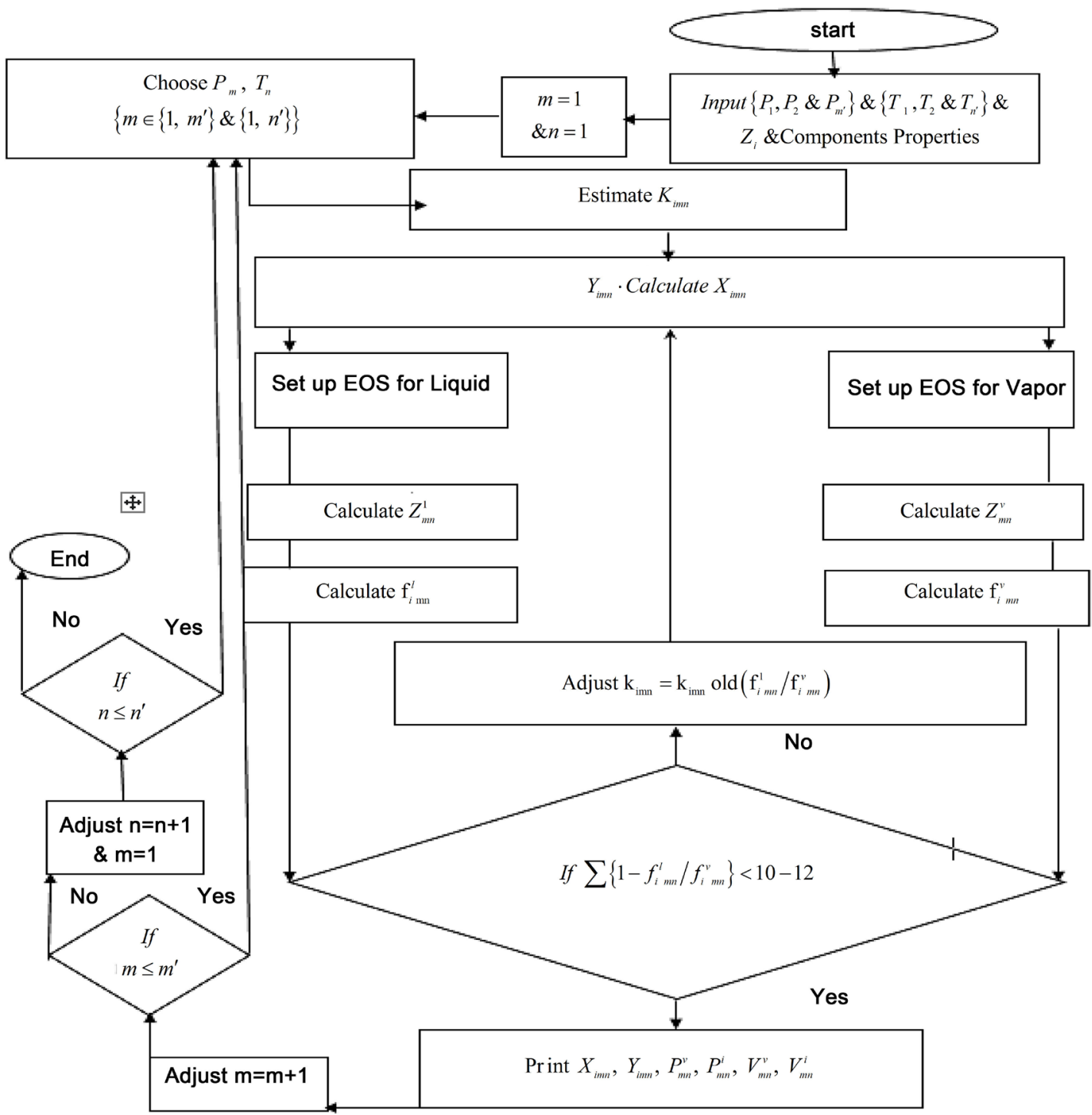

Figure 1. Separator design algorithm. 




Figure 2. Optimization algorithm.

weight were observed.

Finally, by applying some rules that liquid volume must be maximum and liquid density must be minimum in separators, we could calculate optimum pressure and temperature with the help of this easy-to-use the simulator.

\section{Results and Discussion}

The simulation occurred with the help of the software below:

a) Aspen Plus b) Aspen Hysys c) PVTi d) Matlab

For analysis, we utilized from a data-set of gas condensate reservoir with $370 \mathrm{k}$ temperature and 250 bar pressure and composition like as Table 1. (Note that $\gamma \mathrm{C} 7+=0.8 \& \mathrm{MWC} 7+=180)$

\subsection{Aspen Plus Analysis}

We did calculations in three parts with the Aspen Plus analysis.

Part 1: Simulation with one separator and one stock tank as Figure 3 and 
Table 1. Composition and mole percent of components.

\begin{tabular}{ccc}
\hline No. & Component (-) & Mol percent (-) \\
\hline 1 & N2 & 0.29 \\
2 & CO2 & 1.72 \\
3 & C1 & 79.14 \\
4 & C2 & 7.48 \\
5 & C3 & 3.29 \\
6 & IC4 & 0.51 \\
7 & NC4 & 1.25 \\
8 & IC5 & 0.36 \\
9 & NC5 & 0.55 \\
10 & C6 & 0.61 \\
11 & C7 + & 4.8 \\
\hline
\end{tabular}



Figure 3. Simulation with one separator and one stock tank tank schematic in Aspen Plus analysis.

analysis results are as Table 2.

Part 2: Simulation with two separators and one stock tank as Figure 4 and analysis results are as Table 3.

Part 3: Simulation with three separators and one stock tank as Figure 5 and analysis results are as Table 4 .

As results, we can see that by increasing in the separators number, the stock tank liquid volume is increased and the stock tank liquid density is decreased as shown in Figure 6(a) and Figure 6(b).

As shown in Figure above, by increasing the separator number from one separator to three separators, the stage of separation process is increased and the separation occurs in a high quality situation. Therefore, the stock tank liquid volume is increased and the stock tank liquid density is decreased, respectively.

\subsection{Aspen Hysys Analysis}

We did calculations in three parts with the Aspen Hysys analysis.

Part 1: Simulation with one separator and one stock tank as Figure 7 and analysis results are as Table 5 .

Part 2: Simulation with two separators and one stock tank as Figure 8 and analysis results are as Table 6 . 
Table 2. Aspen Plus analysis results with one separator and one stock tank.

\begin{tabular}{|c|c|c|c|c|c|c|}
\hline & & Feed & G1 & G2 & L1 & L2 \\
\hline C7+ Flow & $(\mathrm{kmol} / \mathrm{hr})$ & 4.8 & $3.33 \mathrm{E}-03$ & $8.30 \mathrm{E}-04$ & 4.796675 & 4.795845 \\
\hline N2 Flow & $(\mathrm{kmol} / \mathrm{hr})$ & 0.29 & 0.285368 & $4.62 \mathrm{E}-03$ & $4.63 \mathrm{E}-03$ & $9.46 \mathrm{E}-06$ \\
\hline CO2 Flow & $(\mathrm{kmol} / \mathrm{hr})$ & 1.72 & 1.539227 & 0.176727 & 0.180773 & $4.05 \mathrm{E}-03$ \\
\hline C1 Flow & $(\mathrm{kmol} / \mathrm{hr})$ & 79.14 & 76.17237 & 2.950285 & 2.96763 & 0.017344 \\
\hline C2 Flow & $(\mathrm{kmol} / \mathrm{hr})$ & 7.48 & 6.47294 & 0.974496 & 1.00706 & 0.032564 \\
\hline C3 Flow & $(\mathrm{kmol} / \mathrm{hr})$ & 3.29 & 2.31863 & 0.867913 & 0.971371 & 0.103458 \\
\hline IC4 Flow & $(\mathrm{kmol} / \mathrm{hr})$ & 0.51 & 0.274789 & 0.180572 & 0.235211 & 0.054639 \\
\hline NC4 Flow & $(\mathrm{kmol} / \mathrm{hr})$ & 1.25 & 0.588138 & 0.463662 & 0.661862 & 0.1982 \\
\hline IC5 Flow & $(\mathrm{kmol} / \mathrm{hr})$ & 0.36 & 0.111993 & 0.120292 & 0.248008 & 0.127715 \\
\hline NC5 Flow & $(\mathrm{kmol} / \mathrm{hr})$ & 0.55 & 0.142699 & 0.166146 & 0.407301 & 0.241155 \\
\hline C6 Flow & $(\mathrm{kmol} / \mathrm{hr})$ & 0.61 & 0.073492 & 0.093216 & 0.536508 & 0.443292 \\
\hline Flow $_{\text {тот. }}$ & $(\mathrm{kmol} / \mathrm{hr})$ & 100 & 87.98297 & 5.998763 & 12.01703 & $\underline{6.018266}$ \\
\hline $\mathrm{T}$ & $\left({ }^{\circ} \mathrm{C}\right)$ & 96.85 & 25 & 25 & 25 & 25 \\
\hline $\mathbf{P}$ & (bar) & 250 & 57 & 1 & 57 & 1 \\
\hline Fraction $_{\text {VAP. }}$ & $(-)$ & 0.828596 & 1 & 1 & 0 & 0 \\
\hline Fraction $_{\text {LIQ. }}$ & $(-)$ & 0.171404 & 0 & 0 & 1 & 1 \\
\hline Fraction $_{\text {sol. }}$ & $(-)$ & 0 & 0 & 0 & 0 & 0 \\
\hline $\mathrm{E}$ & $(\mathrm{cal} / \mathrm{mol})$ & -22877.4 & -19958.1 & -24034.8 & -50608.3 & -74755.1 \\
\hline $\mathrm{E}$ & $(\mathrm{cal} / \mathrm{gm})$ & -814.732 & -1051.42 & -762.422 & -534.474 & -474.193 \\
\hline E & $(\mathrm{cal} / \mathrm{sec})$ & $-6.35 \mathrm{E}+05$ & $-4.88 \mathrm{E}+05$ & $-4.00 \mathrm{E}+04$ & $-1.69 \mathrm{E}+05$ & $-1.25 \mathrm{E}+05$ \\
\hline$S$ & (cal/mol-k) & -45.8335 & -30.3582 & -39.2842 & -158.595 & -263.467 \\
\hline$S$ & (cal/gm-k) & -1.63227 & -1.59931 & -1.24616 & -1.67492 & -1.67125 \\
\hline $\mathbf{P}$ & $(\mathrm{mol} / \mathrm{cc})$ & $8.81 \mathrm{E}-03$ & $2.73 \mathrm{E}-03$ & $4.07 \mathrm{E}-05$ & $7.04 \mathrm{E}-03$ & $4.90 \mathrm{E}-03$ \\
\hline $\mathbf{P}$ & $(\mathrm{gm} / \mathrm{cc})$ & 0.247364 & 0.051802 & $1.28 \mathrm{E}-03$ & 0.666511 & $\underline{0.772773}$ \\
\hline $\mathrm{MW}_{\mathrm{AV}}$. & $(\mathrm{gm} / \mathrm{mol})$ & 28.07965 & 18.98205 & 31.5243 & 94.68798 & 157.647 \\
\hline $\mathrm{V}_{\mathrm{L}}$ & $(\mathrm{cc} / \mathrm{min})$ & 111.8395 & 84.24125 & 7.268958 & 27.59826 & 20.3293 \\
\hline
\end{tabular}

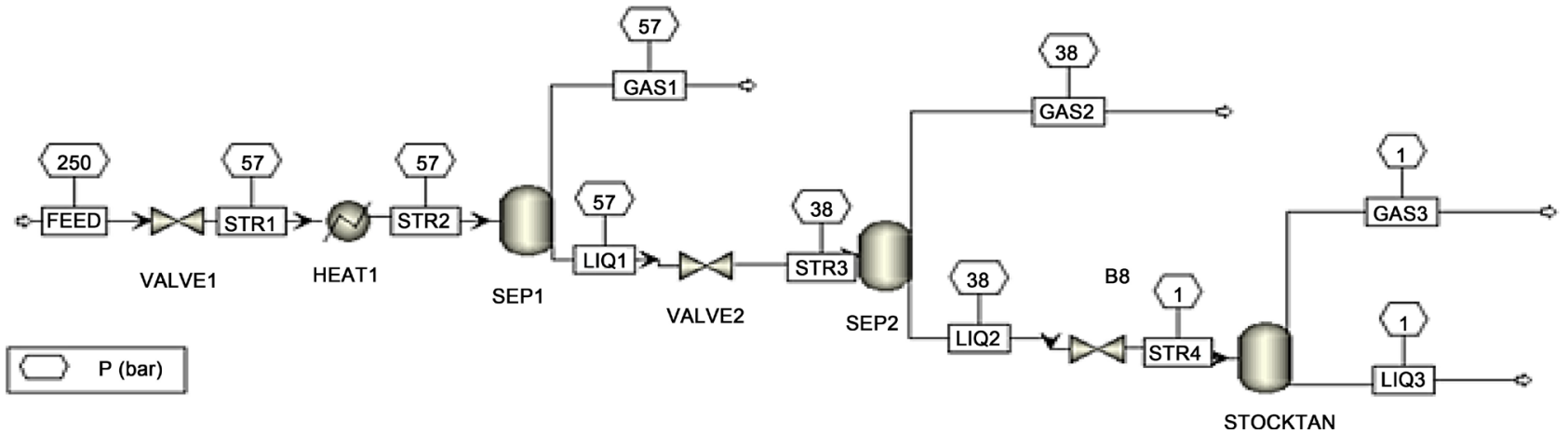

Figure 4. Simulation with two separators and one stock tank schematic in Aspen Plus analysis. 
Table 3. Aspen Plus analysis results with two separators and one stock tank.

\begin{tabular}{|c|c|c|c|c|c|c|c|c|}
\hline & & Feed & G1 & G2 & G3 & L1 & L2 & L3 \\
\hline C7+ Flow & $(\mathrm{kmol} / \mathrm{hr})$ & 4.8 & $3.33 \mathrm{E}-03$ & $2.99 \mathrm{E}-05$ & $5.99 \mathrm{E}-04$ & 4.796675 & 4.796645 & 4.796046 \\
\hline N2 Flow & $(\mathrm{kmol} / \mathrm{hr})$ & 0.29 & 0.285368 & $2.92 \mathrm{E}-03$ & $1.71 \mathrm{E}-03$ & $4.63 \mathrm{E}-03$ & $1.71 \mathrm{E}-03$ & $4.87 \mathrm{E}-06$ \\
\hline CO2 Flow & $(\mathrm{kmol} / \mathrm{hr})$ & 1.72 & 1.539227 & 0.031015 & 0.145148 & $1.81 \mathrm{E}-01$ & 0.149758 & $4.61 \mathrm{E}-03$ \\
\hline C1 Flow & $(\mathrm{kmol} / \mathrm{hr})$ & 79.14 & 76.17237 & 1.187863 & 1.765283 & 2.96763 & 1.779767 & 0.014484 \\
\hline C2 Flow & $(\mathrm{kmol} / \mathrm{hr})$ & 7.48 & 6.47294 & 0.130382 & 0.837625 & 1.00706 & 0.876678 & 0.039054 \\
\hline C3 Flow & $(\mathrm{kmol} / \mathrm{hr})$ & 3.29 & 2.31863 & 0.046648 & 0.792982 & 0.971371 & 0.924722 & 0.131741 \\
\hline IC4 Flow & $(\mathrm{kmol} / \mathrm{hr})$ & 0.51 & 0.274789 & $5.28 \mathrm{E}-03$ & 0.161714 & 0.235211 & 0.229926 & 0.068212 \\
\hline NC4 Flow & $(\mathrm{kmol} / \mathrm{hr})$ & 1.25 & 0.588138 & 0.011132 & 0.407847 & 0.661862 & 0.650731 & 0.242884 \\
\hline IC5 Flow & $(\mathrm{kmol} / \mathrm{hr})$ & 0.36 & 0.111993 & $1.98 \mathrm{E}-03$ & 0.099258 & 0.248008 & 0.246024 & 0.146766 \\
\hline NC5 Flow & $(\mathrm{kmol} / \mathrm{hr})$ & 0.55 & 0.142699 & $2.50 \mathrm{E}-03$ & 0.134063 & 0.407301 & 0.404805 & 0.270743 \\
\hline C6 Flow & $(\mathrm{kmol} / \mathrm{hr})$ & 0.61 & 0.073492 & $1.18 \mathrm{E}-03$ & 0.070246 & 0.536508 & 0.535324 & 0.465077 \\
\hline Flow $_{\text {тот. }}$ & $(\mathrm{kmol} / \mathrm{hr})$ & 100 & 87.98297 & 1.420939 & 4.41647 & 12.01703 & 10.59609 & $\underline{6.17962}$ \\
\hline $\mathrm{T}$ & $\left({ }^{\circ} \mathrm{C}\right)$ & 96.85 & 25 & 25 & 25 & 25 & 25 & 25 \\
\hline $\mathbf{P}$ & (bar) & 250 & 57 & 38 & 1 & 57 & 38 & 1 \\
\hline Fraction $_{\mathrm{VAP}}$ & $(-)$ & 0.828596 & 1 & 1 & 1 & 0 & 0 & 0 \\
\hline Fraction $_{\text {LIQ }}$ & $(-)$ & 0.171404 & 0 & 0 & 0 & 1 & 1 & 1 \\
\hline Fraction $_{\text {sol. }}$ & $(-)$ & 0 & 0 & 0 & 0 & 0 & 0 & 0 \\
\hline E & $(\mathrm{cal} / \mathrm{mol})$ & -22877.4 & -19958.1 & -20323 & -24992.9 & -50608.3 & -54557.9 & -73795.8 \\
\hline E & (cal/gm) & -814.732 & -1051.42 & -1036.32 & -730.942 & -534.474 & -520.81 & -475.531 \\
\hline E & $(\mathrm{cal} / \mathrm{sec})$ & $-6.35 E+05$ & $-4.88 \mathrm{E}+05$ & -8021.61 & -30661.2 & $-1.69 \mathrm{E}+05$ & $-1.61 \mathrm{E}+05$ & $-1.27 \mathrm{E}+05$ \\
\hline$S$ & (cal/mol-k) & -45.8335 & -30.3582 & -29.9179 & -43.1607 & -158.595 & -175.176 & -259.43 \\
\hline$S$ & (cal/gm-k) & -1.63227 & -1.59931 & -1.52558 & -1.26228 & -1.67492 & -1.67223 & -1.67173 \\
\hline $\mathbf{P}$ & $(\mathrm{mol} / \mathrm{cc})$ & $8.81 \mathrm{E}-03$ & $2.73 \mathrm{E}-03$ & $1.73 \mathrm{E}-03$ & $4.07 \mathrm{E}-05$ & $7.04 \mathrm{E}-03$ & $6.63 \mathrm{E}-03$ & $4.96 \mathrm{E}-03$ \\
\hline $\mathbf{P}$ & $(\mathrm{gm} / \mathrm{cc})$ & 0.247364 & 0.051802 & 0.034017 & $1.39 \mathrm{E}-03$ & 0.666511 & 0.694998 & $\underline{0.770258}$ \\
\hline $\mathrm{MW}_{\mathrm{AV}}$. & $(\mathrm{gm} / \mathrm{mol})$ & 28.07965 & 18.98205 & 19.61078 & 34.19267 & 94.68798 & 104.7559 & 155.1862 \\
\hline $\mathrm{V}_{\mathrm{L}}$ & $(\mathrm{cc} / \mathrm{min})$ & 111.8395 & 84.24125 & 1.381344 & 5.600417 & 27.59826 & 26.21692 & 20.6165 \\
\hline
\end{tabular}

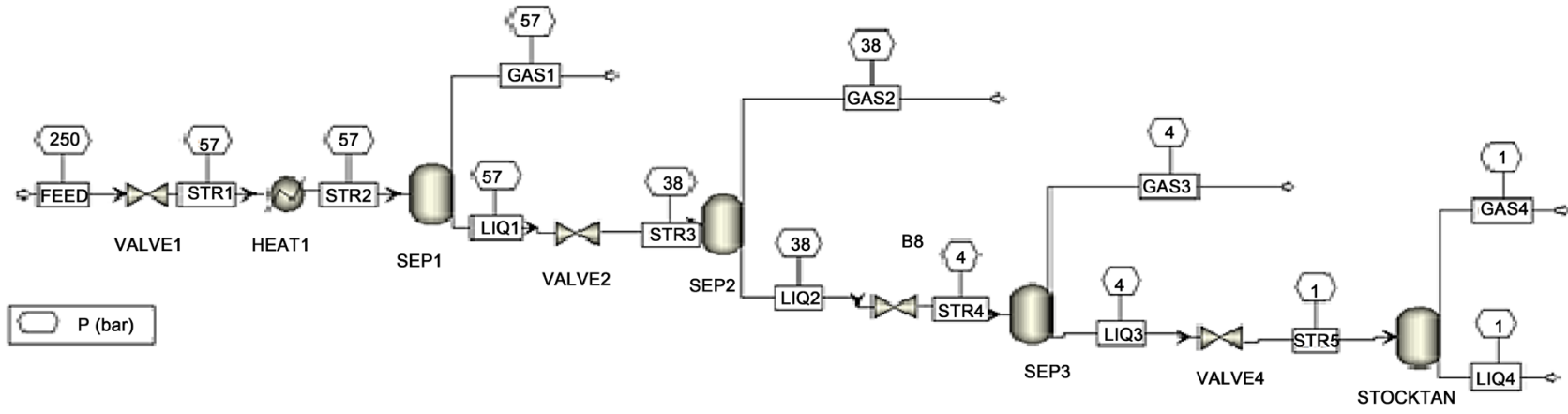

Figure 5. Simulation with three separators and one stock tank schematic in Aspen Plus analysis. 
Table 4. Aspen Plus analysis results with three separators and one stock tank.

\begin{tabular}{|c|c|c|c|c|c|c|c|c|c|}
\hline & & Feed & G1 & G3 & G4 & L1 & L2 & L3 & L4 \\
\hline C7+ Flow & $(\mathrm{kmol} / \mathrm{hr})$ & 4.8 & $3.33 \mathrm{E}-03$ & $1.15 \mathrm{E}-04$ & $8.35 \mathrm{E}-05$ & 4.796675 & 4.796645 & 4.796529 & 4.796446 \\
\hline N2 Flow & $(\mathrm{kmol} / \mathrm{hr})$ & $2.90 \mathrm{E}-01$ & $2.85 \mathrm{E}-01$ & $1.68 \mathrm{E}-03$ & $3.07 \mathrm{E}-05$ & $4.63 \mathrm{E}-03$ & $1.71 \mathrm{E}-03$ & $3.14 \mathrm{E}-05$ & $6.41 \mathrm{E}-07$ \\
\hline CO2 Flow & $(\mathrm{kmol} / \mathrm{hr})$ & 1.72 & 1.539227 & $1.25 \mathrm{E}-01$ & 0.020164 & $1.81 \mathrm{E}-01$ & 0.149758 & $2.48 \mathrm{E}-02$ & $4.61 \mathrm{E}-03$ \\
\hline C1 Flow & $(\mathrm{kmol} / \mathrm{hr})$ & 79.14 & 76.17237 & 1.689443 & 0.085201 & 2.96763 & 1.779767 & $9.03 \mathrm{E}-02$ & $5.12 \mathrm{E}-03$ \\
\hline C2 Flow & $(\mathrm{kmol} / \mathrm{hr})$ & 7.48 & 6.47294 & 0.675978 & 0.149642 & 1.00706 & 0.876678 & 0.200701 & 0.051059 \\
\hline C3 Flow & $(\mathrm{kmol} / \mathrm{hr})$ & 3.29 & 2.31863 & 0.454155 & 0.212735 & 0.971371 & 0.924722 & 0.470568 & 0.257833 \\
\hline IC4 Flow & $(\mathrm{kmol} / \mathrm{hr})$ & $5.10 \mathrm{E}-01$ & 0.274789 & 0.063909 & 0.040708 & 0.235211 & 0.229926 & 0.166017 & 0.12531 \\
\hline NC4 Flow & $(\mathrm{kmol} / \mathrm{hr})$ & 1.25 & 0.588138 & 0.140246 & 0.095643 & 0.661862 & 0.650731 & 0.510485 & 0.414842 \\
\hline IC5 Flow & $(\mathrm{kmol} / \mathrm{hr})$ & $3.60 \mathrm{E}-01$ & 0.111993 & 0.024874 & 0.018816 & 0.248008 & 0.246024 & 0.22115 & 0.202334 \\
\hline NC5 Flow & $(\mathrm{kmol} / \mathrm{hr})$ & $5.50 \mathrm{E}-01$ & 0.142699 & 0.031085 & 0.023904 & 0.407301 & 0.404805 & 0.37372 & 0.349817 \\
\hline C6 Flow & $(\mathrm{kmol} / \mathrm{hr})$ & $6.10 \mathrm{E}-01$ & 0.073492 & 0.013367 & 0.010629 & 0.536508 & 0.535324 & 0.521956 & 0.511327 \\
\hline Flow $_{\text {тот. }}$ & $(\mathrm{kmol} / \mathrm{hr})$ & 100 & 87.98297 & 3.219836 & 0.657556 & 12.01703 & 10.59609 & 7.376254 & $\underline{6.718698}$ \\
\hline $\mathrm{T}$ & $\left({ }^{\circ} \mathrm{C}\right)$ & 96.85 & 25 & 25 & 25 & 25 & 25 & 25 & 25 \\
\hline $\mathbf{P}$ & (bar) & 250 & 57 & 4 & 1 & 57 & 38 & 4 & 1 \\
\hline Fraction $_{\text {VAP. }}$ & $(-)$ & 0.828596 & 1 & 1 & 1 & 0 & 0 & 0 & 0 \\
\hline Fraction $_{\text {LIQ }}$ & $(-)$ & 0.171404 & 0 & 0 & 0 & 1 & 1 & 1 & 1 \\
\hline Fraction $_{\text {SOL. }}$ & $(-)$ & 0 & 0 & 0 & 0 & 0 & 0 & 0 & 0 \\
\hline E & $(\mathrm{cal} / \mathrm{mol})$ & -22877.4 & -19958.1 & -23499.5 & -27201.4 & -50608.3 & -54557.9 & -67255.3 & -70822.1 \\
\hline E & (cal/gm) & -814.732 & -1051.42 & -839.969 & -637.115 & -534.474 & -520.81 & -486.402 & -479.743 \\
\hline E & $(\mathrm{cal} / \mathrm{sec})$ & $-6.35 E+05$ & $-4.88 \mathrm{E}+05$ & -21018 & -4968.46 & $-1.69 \mathrm{E}+05$ & $-1.61 \mathrm{E}+05$ & $-1.38 \mathrm{E}+05$ & $-1.32 \mathrm{E}+05$ \\
\hline$S$ & (cal/mol-k) & -45.8335 & -30.3582 & -36.0418 & -56.9177 & -158.595 & -175.176 & -231.344 & -247.065 \\
\hline$S$ & (cal/gm-k) & -1.63227 & -1.59931 & -1.28828 & -1.33313 & -1.67492 & -1.67223 & -1.67312 & -1.6736 \\
\hline$\rho$ & $(\mathrm{mol} / \mathrm{cc})$ & $8.81 \mathrm{E}-03$ & $2.73 \mathrm{E}-03$ & $1.66 \mathrm{E}-04$ & $4.09 \mathrm{E}-05$ & $7.04 \mathrm{E}-03$ & $6.63 \mathrm{E}-03$ & $5.43 \mathrm{E}-03$ & $5.16 \mathrm{E}-03$ \\
\hline$\rho$ & $(\mathrm{gm} / \mathrm{cc})$ & 0.247364 & $5.18 \mathrm{E}-02$ & $4.63 \mathrm{E}-03$ & $1.75 \mathrm{E}-03$ & 0.666511 & 0.694998 & 0.751315 & $\underline{0.762066}$ \\
\hline $\mathrm{MW}_{\mathrm{AV}}$. & $(\mathrm{gm} / \mathrm{mol})$ & 28.07965 & 18.98205 & 27.97669 & 42.69463 & 94.68798 & 104.7559 & 138.271 & 147.625 \\
\hline $\mathrm{V}_{\mathrm{L}}$ & $(\mathrm{cc} / \mathrm{min})$ & 111.8395 & 84.24125 & 3.715306 & 0.948889 & 27.59826 & 26.21692 & 22.50161 & 21.55272 \\
\hline
\end{tabular}

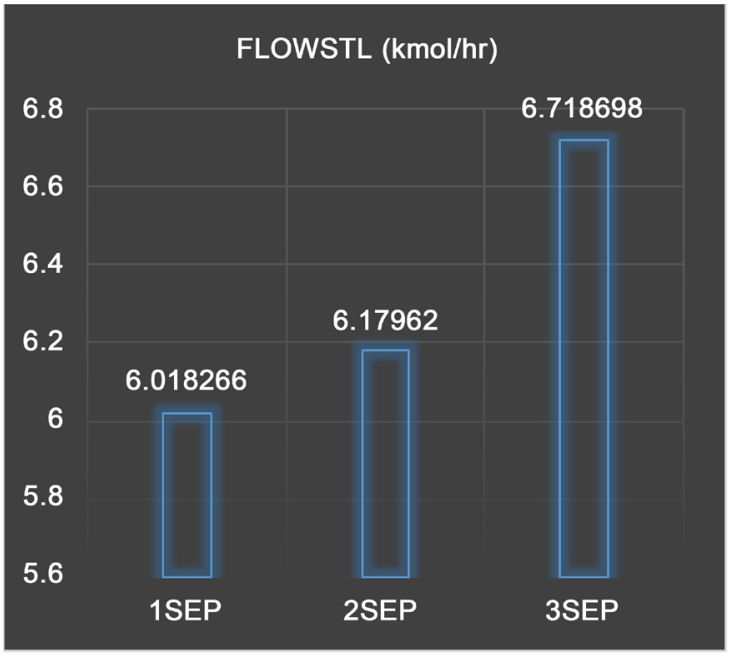

(a)

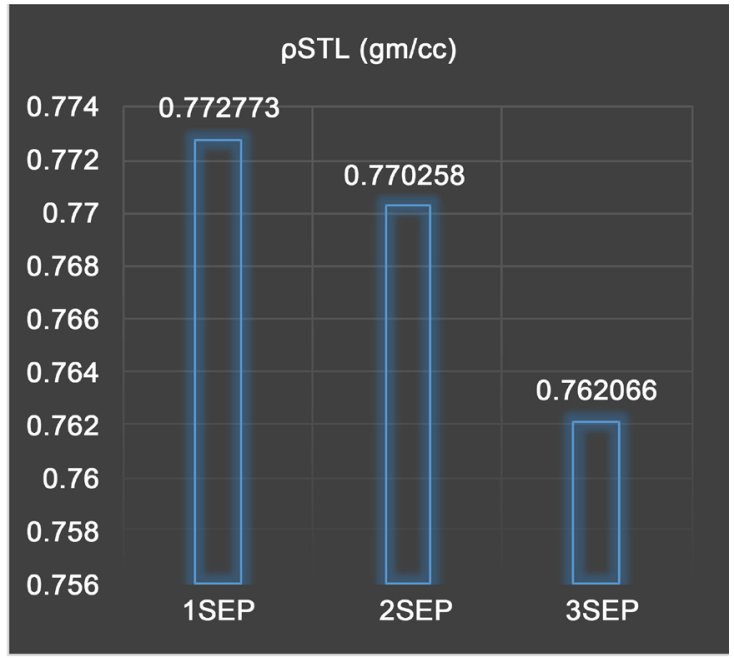

(b)

Figure 6. (a) Stock tank liquid volume increasing; (b) Stock tank liquid density decreasing by separators number increasing in Aspen Plus analysis. 


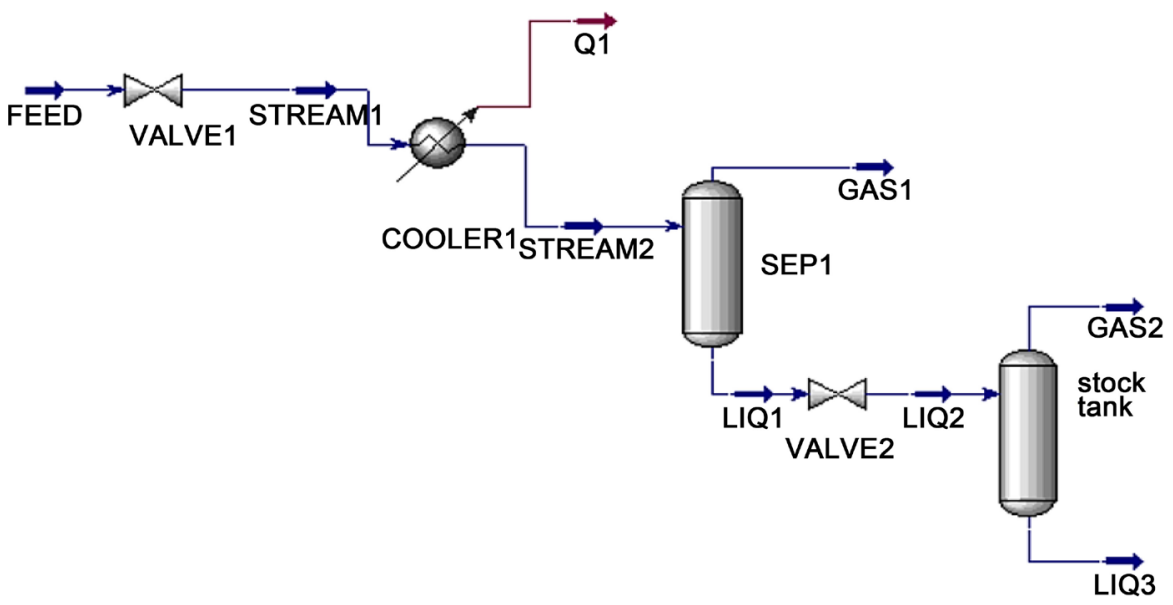

Figure 7. Simulation with one separator and one stock tank tank schematic in Aspen Hysys analysis.

Table 5. Aspen Hysys analysis results with one separator and one stock tank.

\begin{tabular}{|c|c|c|c|c|c|}
\hline & & Feed & G1 & L1 & L3 \\
\hline Flow fraction $n_{\text {VAP. }}$ & $(-)$ & 0.863477 & 1 & 0 & 0 \\
\hline $\mathrm{T}$ & $\left({ }^{\circ} \mathrm{C}\right)$ & 96.85 & 26.15107 & 26.15107 & 6.440161 \\
\hline $\mathbf{P}$ & (kpa) & 25000 & 7000 & 7000 & 100 \\
\hline Flow $_{\text {тот. }}$ & $(\mathrm{kmol} / \mathrm{hr})$ & 100 & 88.42481 & 11.57519 & 6.276834 \\
\hline Flow $_{\text {тот. }}$ & $(\mathrm{kg} / \mathrm{hr})$ & 2807.982 & 1688.545 & 1119.436 & 965.2965 \\
\hline $\mathrm{V}_{\mathrm{L}}$ & $(\mathrm{m} 3 / \mathrm{hr})$ & 6.702509 & 5.085935 & 1.616573 & 1.24514 \\
\hline $\mathrm{Q}$ & $(\mathrm{kj} / \mathrm{hr})$ & $9,783,158$ & $7,468,622$ & $2,661,763$ & $2,143,982$ \\
\hline
\end{tabular}

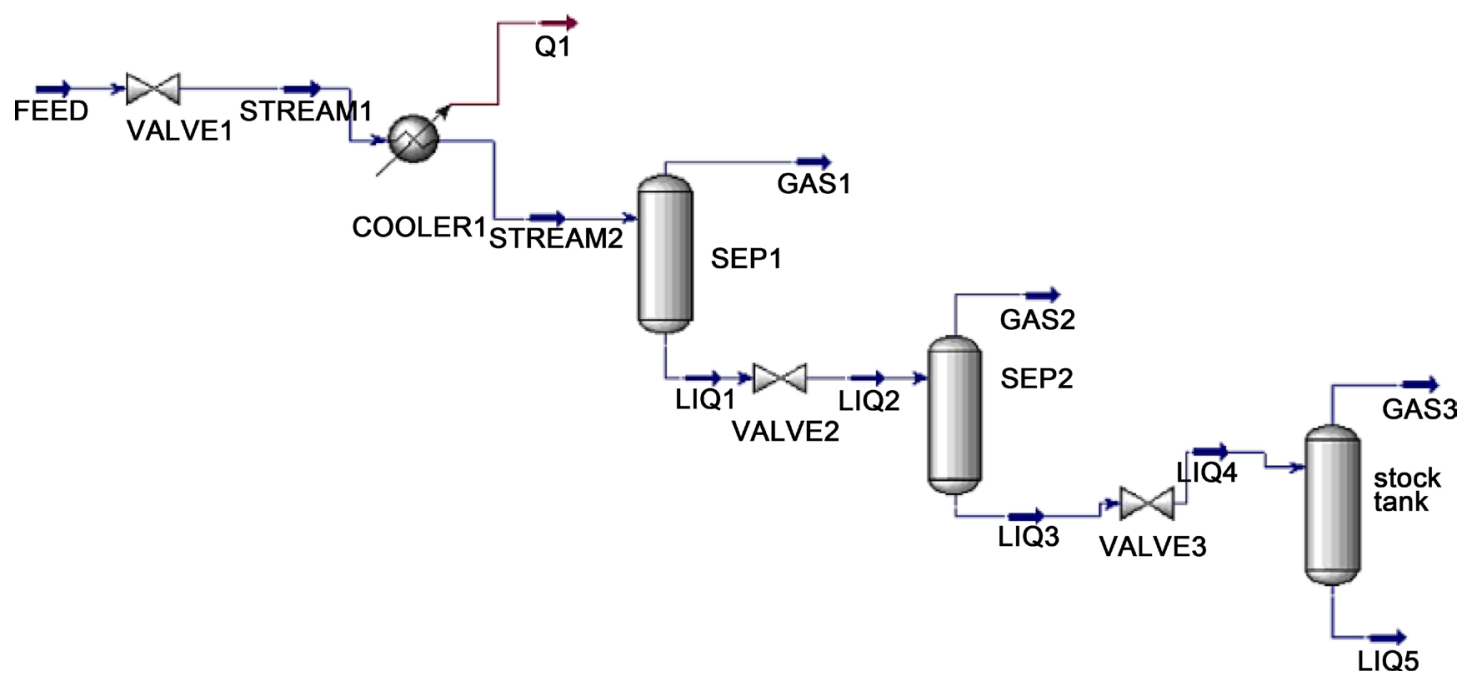

Figure 8. Simulation with two separators and one stock tank tank schematic in Aspen Hysys analysis.

Part 3: Simulation with three separators and one stock tank as Figure 9 and analysis results are as Table 7.

As results, we can see that by increasing in the separators number, the stock tank liquid volume is increased and the stock tank liquid density is decreased as shown in Figure 10(a) and Figure 10(b). 
Table 6. Aspen Hysys analysis results with two separators and one stock tank.

\begin{tabular}{|c|c|c|c|c|c|c|c|c|}
\hline & & Feed & G1 & G2 & G3 & $\mathrm{L} 1$ & L3 & L5 \\
\hline Flow fraction ${ }_{\text {VAP. }}$ & $(-)$ & 0.863477 & 1 & 1 & 1 & 0 & 0 & 0 \\
\hline $\mathrm{T}$ & $\left({ }^{\circ} \mathrm{C}\right)$ & 96.85 & 26.15107 & 24.30809 & 8.421311 & 26.15107 & 24.30809 & 8.421311 \\
\hline $\mathbf{P}$ & (kpa) & 25000 & 7000 & 4000 & 100 & 7000 & 4000 & 100 \\
\hline Flow $_{\text {тот. }}$ & $(\mathrm{kmol} / \mathrm{hr})$ & 100 & 88.42481 & 1.682519 & 3.453154 & 11.57519 & 9.892669 & $\underline{6.439515}$ \\
\hline Flow $_{\text {тот. }}$ & $(\mathrm{kg} / \mathrm{hr})$ & 2807.982 & 1688.545 & 33.16979 & 111.4076 & 1119.436 & 1086.267 & 974.859 \\
\hline $\mathrm{V}_{\mathrm{L}}$ & $\left(\mathrm{m}^{3} / \mathrm{hr}\right)$ & 6.702509 & 5.085935 & $9.83 \mathrm{E}-02$ & 0.256468 & 1.616573 & 1.518244 & 1.261776 \\
\hline Q & $(\mathrm{kj} / \mathrm{hr})$ & $-9,783,158$ & $-7,468,622$ & $-144,383$ & $-352,291$ & $-2,661,763$ & $-2,517,380$ & $-2,165,089$ \\
\hline
\end{tabular}

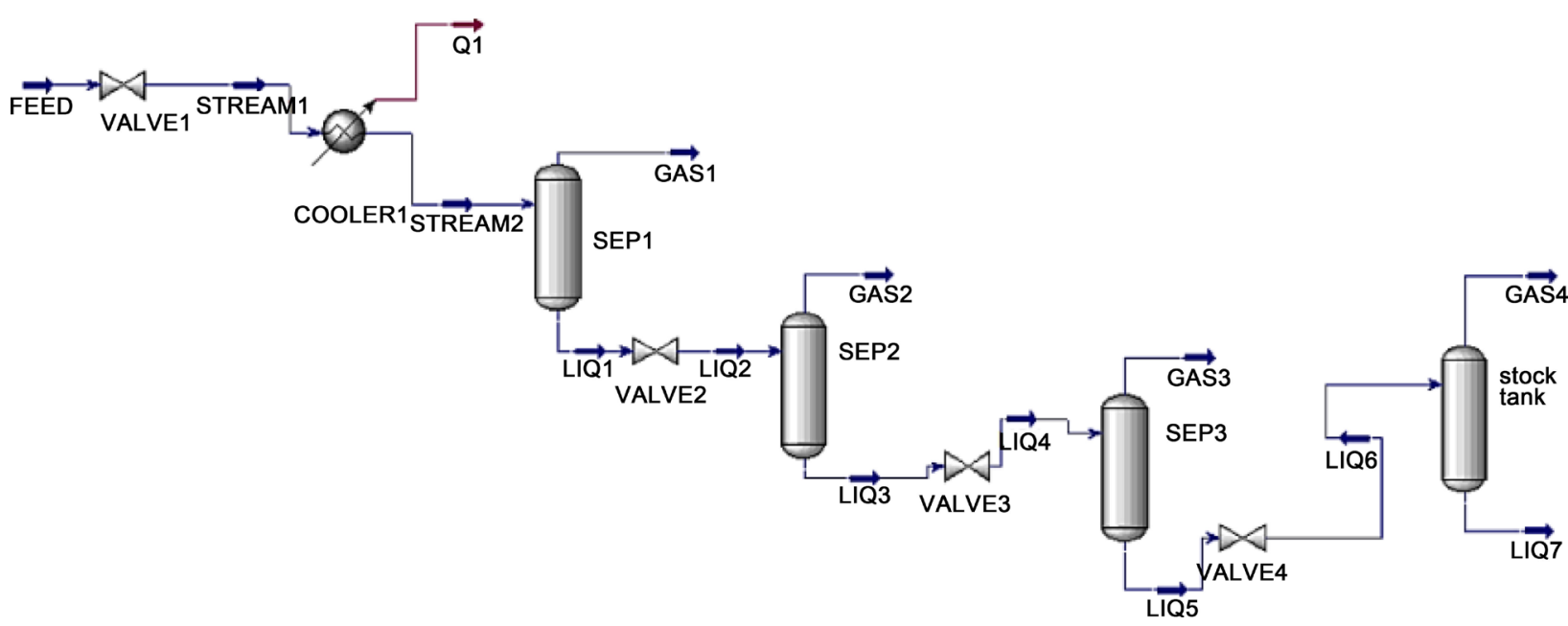

Figure 9. Simulation with three separators and one stock tank tank schematic in Aspen Hysys analysis.

Table 7. Aspen Hysys analysis results with three separators and one stock tank.

\begin{tabular}{|c|c|c|c|c|c|c|c|c|}
\hline & & Feed & G1 & G2 & L1 & L3 & L5 & L7 \\
\hline Flow fraction ${ }_{\mathrm{VAP}}$ & $(-)$ & 0.863477 & 1 & 1 & 0 & 0 & 0 & 0 \\
\hline $\mathrm{T}$ & $\left({ }^{\circ} \mathrm{C}\right)$ & 96.85 & 26.15107 & 24.30809 & 26.15107 & 24.30809 & 21.45056 & 10.80439 \\
\hline $\mathbf{P}$ & (kpa) & 25000 & 7000 & 4000 & 7000 & 4000 & 1500 & 100 \\
\hline Flow $_{\text {тот. }}$ & $(\mathrm{kmol} / \mathrm{hr})$ & 100 & 88.42481 & 1.682519 & 11.57519 & 9.892669 & 8.452935 & 6.673252 \\
\hline Flow $_{\text {тот. }}$ & $(\mathrm{kg} / \mathrm{hr})$ & 2807.982 & 1688.545 & 33.16979 & 1119.436 & 1086.267 & 1054.275 & 988.1731 \\
\hline $\mathrm{V}_{\mathrm{L}}$ & $\left(\mathrm{m}^{3} / \mathrm{hr}\right)$ & 6.702509 & 5.085935 & $9.83 \mathrm{E}-02$ & 1.616573 & 1.518244 & 1.428958 & 1.285273 \\
\hline $\mathbf{Q}$ & $(\mathrm{kj} / \mathrm{hr})$ & -9783158 & -7468622 & -144383 & -2661763 & -2517380 & -2386674 & -2195199 \\
\hline
\end{tabular}

As shown in Figure above, by increasing the separator number from one separator to three separators, the stage of separation process is increased and the separation occurs in a high quality situation. Therefore, the stock tank liquid volume is increased and the stock tank liquid density is decreased, respectively.

\subsection{PVTi Analysis}

We did calculations in one part with the PVTi analysis. 


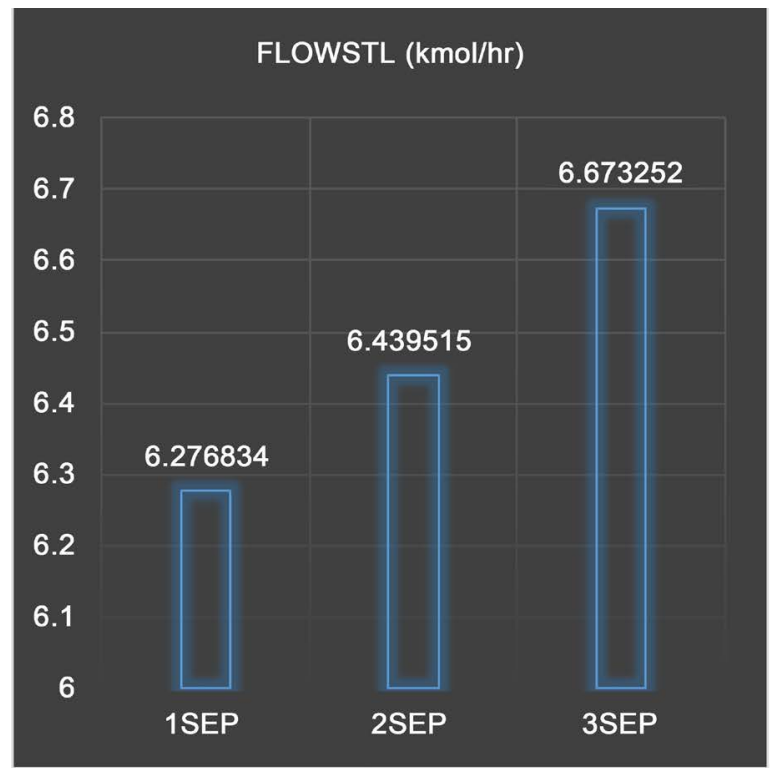

(a)

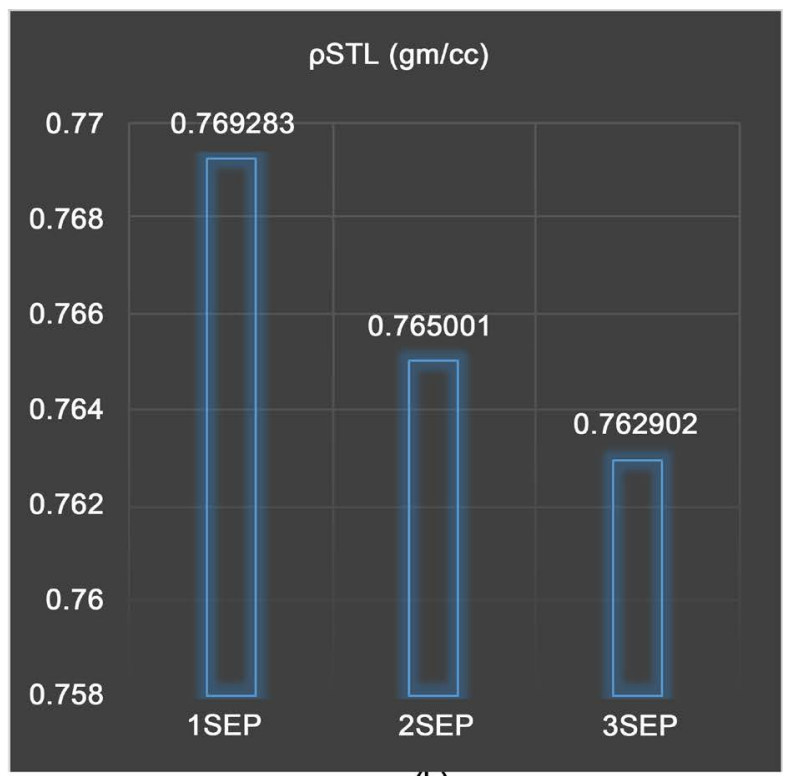

(b)

Figure 10. (a) Stock tank liquid volume increasing; (b) Stock tank liquid density decreasing by separators number increasing in Aspen Hysys analysis.

Table 8. PVTi analysis results with three separators and one stock tank.

\begin{tabular}{|c|c|c|c|c|c|}
\hline & & Sep. 1 & Sep. 2 & Sep. 3 & S.T. \\
\hline Mol fraction $n_{\mathrm{VAP}}$ & $(-)$ & 0.888 & 0.9039 & 0.928 & 0.928 \\
\hline Mol fraction $_{\text {LIQ. }}$ & $(-)$ & 0.112 & 0.0961 & 0.072 & $\underline{0.072}$ \\
\hline $\mathrm{V}_{\mathrm{v}}$ & $\left(\mathrm{Sm}^{3}\right)$ & 21.0368 & 21.4149 & 21.9865 & 21.9865 \\
\hline $\mathrm{V}_{\mathrm{L}}$ & $\left(\mathrm{m}^{3}\right)$ & 0.0158 & 0.0148 & 0.0131 & 0.013 \\
\hline GOR & $\left(\mathrm{Sm}^{3} / \mathrm{m}^{3}\right)$ & 1328.286 & 1442.772 & 1677.038 & 1677.038 \\
\hline $\mathbf{B}_{\mathrm{O}}$ & $\left(\mathrm{Rm}^{3} / \mathrm{Sm}^{3}\right)$ & 1.2144 & 1.1382 & 1.0053 & 1.0053 \\
\hline$\rho_{\mathrm{V}}$ & $\left(\mathrm{kg} / \mathrm{m}^{3}\right)$ & 54.6194 & 34.7169 & 12.375 & 0.8276 \\
\hline$\rho_{\mathrm{L}}$ & $\left(\mathrm{kg} / \mathrm{m}^{3}\right)$ & 704.8399 & 724.8136 & 757.6122 & $\underline{761.6396}$ \\
\hline $\mathrm{MW}_{\mathrm{AV} . \mathrm{v}}$ & $(\mathrm{kgm} / \mathrm{Kmol})$ & 19.0365 & 19.148 & 19.54 & 19.54 \\
\hline $\mathrm{MW}_{\mathrm{AV} . \mathrm{L}}$ & $(\mathrm{kgm} / \mathrm{Kmol})$ & 99.6357 & 111.975 & 138.0461 & 138.0461 \\
\hline $\mathrm{T}$ & (k) & 298.15 & 298.15 & 298.15 & 288.7056 \\
\hline $\mathbf{P}$ & (bar) & 60 & 40 & 15 & 1.0132 \\
\hline
\end{tabular}

Simulation with three separators and one stock tank was done and the simulation results are as Table 8 .

\subsection{Matlab Analysis}

We did the calculations with the help of the two parameters Peng-Robinson equation of state (PR EOS) as shown in Equations (1) through (8) [18]:

$$
P=\frac{R T}{V-b}-\frac{a_{c} \alpha}{V(V+b)+b(V-b)}
$$




$$
\begin{gathered}
a_{c}=0.457235 \frac{R^{2} T_{c}^{2}}{P_{c}} \\
b=0.077796 \frac{R T_{c}}{P_{c}} \\
m=0.3796+1.485 \omega-0.1644 \omega^{2}+0.01667 \omega^{3} \\
A=\frac{a P}{(R T)^{2}} \\
B=\frac{b P}{R T} \\
z^{3}-(1-B) z^{2}+\left(A-2 B-3 B^{2}\right) z-\left(A B-B^{2}-B^{3}\right)=0 \\
\ln \varnothing=(z-1)-\ln (z-B)+\frac{A}{2 B \sqrt{2}} \ln \frac{z+(1-\sqrt{2}) B}{z+(1+\sqrt{2}) B}
\end{gathered}
$$

where $P, V, T, R, a_{\mathcal{c}} b, \alpha, P_{\mathcal{c}}, T_{\mathcal{c}} \omega, \varphi$, and $z$ are the pressure, volume, temperature, universal gas constant, real gas correction factor due to the intermolecular forces, real gas correction factor due to the gas molecular size, temperature-dependent parameter, critical pressure, critical temperature, acentric factor, fugacity coefficient and compressibility factor, respectively.

Equilibrium ratio $\left(k_{i}\right)$ was calculated with the help of the Wilson Correlation as shown in Equation (9) [19] [20]:

$$
k_{i}=\left(\frac{P_{c i}}{P}\right) \exp \left(5.37\left(1+\omega_{i}\right)\left(1-\frac{T_{c i}}{T}\right)\right)
$$

Subscript " $I$ " is related to $i$-component in the two-phase solution.

Flash calculations were calculated with the flash calculations equations as shown in Equations ((10) and (12)):

$$
\begin{gathered}
x_{i}=\frac{z_{i}}{1+\left(k_{i}-1\right) n^{v}} \\
y_{i}=\frac{z_{i} k_{i}}{1+\left(k_{i}-1\right) n^{v}} \\
f\left(n^{v}\right)=\sum_{i=1}^{n}\left(y_{i}-x_{i}\right)=\sum_{i=1}^{n} \frac{z_{i}\left(k_{i}-1\right)}{1+\left(k_{i}-1\right) n^{v}}=0
\end{gathered}
$$

where $x_{i}, y_{i}, Z_{i}$, and $n^{V}$ are the mole percent of $\mathrm{i}$-component in the liquid phase, mole percent of $\mathrm{i}$-component in the gas phase, mole percent of $\mathrm{i}$-component in the two-phase solution, and volume percent of gas (vapor) phase, respectively.

We developed a code that is able to calculate equilibrium calculations for any specific data set and also to obtain the optimum parameters with the help of the algorithms as shown in Figure 1 and Figure 2. Input feed was considered as 100 $\mathrm{kmol} / \mathrm{hr}$.

Simulation with three separators and one stock tank was done and simulation results are as Table 9 and mole fraction of each component in both liquid and 
Table 9. Code analysis results with three separators and one stock tank.

\begin{tabular}{cccccc}
\hline & & Sep.1 & Sep.2 & Sep.3 & S.T. \\
\hline Liq. output & $(\mathrm{kmol} / \mathrm{hr})$ & 11.69 & 10.47 & 9.13 & 8.72 \\
$\mathrm{~T}$ & $\left({ }^{\circ} \mathrm{C}\right)$ & 31.85 & 22.85 & 30.85 & 25 \\
$\mathrm{P}$ & $(\mathrm{bar})$ & 63 & 38 & 13 & 1 \\
\hline
\end{tabular}

Table 10. Code analysis of flash calculation for each separator stage.

\begin{tabular}{cccccccccc}
\hline & & Sep.1 & Sep.2 & Sep.3 & S.T. & Sep.1 & Sep.2 & Sep.3 & S.T. \\
\hline Component & $\mathrm{z}_{\mathrm{i}}(-)$ & $\mathrm{x}_{\mathrm{i}}(-)$ & $\mathrm{x}_{\mathrm{i}}(-)$ & $\mathrm{x}_{\mathrm{i}}(-)$ & $\mathrm{x}_{\mathrm{i}}(-)$ & $\mathrm{y}_{\mathrm{i}}(-)$ & $\mathrm{y}_{\mathrm{i}}(-)$ & $\mathrm{y}_{\mathrm{i}}(-)$ & $\mathrm{y}_{\mathrm{i}}(-)$ \\
$\mathrm{N} 2$ & 0.29 & 0.02 & 0.01 & 0 & 0 & 0.33 & 0.15 & 0.05 & 0.01 \\
CO2 & 1.72 & 1.44 & 1.33 & 0.83 & 0.49 & 1.75 & 2.19 & 4.73 & 2.95 \\
C1 & 79.14 & 15.28 & 8.53 & 1.97 & 0.43 & 87.54 & 72.41 & 53.18 & 12.31 \\
C2 & 7.48 & 9.2 & 9.01 & 6.71 & 0.466 & 7.23 & 9.73 & 24.59 & 18.29 \\
C3 & 3.29 & 11.63 & 12.47 & 12.65 & 11.99 & 2.15 & 3.02 & 10.87 & 11.03 \\
IC4 & 0.51 & 2.82 & 3.07 & 3.35 & 3.41 & 0.2 & 0.27 & 1.09 & 1.18 \\
NC4 & 1.25 & 7.7 & 8.42 & 9.31 & 9.62 & 0.37 & 0.51 & 2.09 & 2.31 \\
IC5 & 0.36 & 2.64 & 2.9 & 3.27 & 3.45 & 0.05 & 0.07 & 0.029 & 0.32 \\
NC5 & 0.55 & 4.16 & 4.57 & 5.17 & 5.46 & 0.06 & 0.08 & 0.35 & 0.39 \\
C6 & 0.61 & 4.93 & 5.43 & 6.18 & 6.58 & 0.02 & 0.03 & 0.13 & 0.15 \\
C7+ & 4.8 & 40.18 & 44.26 & 50.55 & 53.93 & 0 & 0 & 0 & 0 \\
\hline
\end{tabular}

gas phases were calculated for each separator stage too as Table 10.

Code analysis in the optimum parameters calculations shows that output liquid volume and density from the third separator or input liquid volume and density into stock tank calculated from the code is higher and lower than calculated from other simulators that are very important issue in petroleum engineering surface facilities. According to the algorithm, calculations of the third separators in a range of pressures and temperatures shown in Figure 11 that is obvious that in what pressure and temperature we have the highest liquid volume and the lowest liquid density, these quantities are optimum quantities.

Finally, we concern on the optimum parameters calculated with the different simulators to do a comparison. Optimum pressure, temperature, and liquid output volume calculated from the different simulators are as Figures 12(a)-(c).

The liquid output from the third separator is very important that is maximum in the code calculations in comparison to other simulators as Figure 13.

\section{Conclusion}

A computer simulator is written to optimize the pressure, temperature, and the number of separators of gas condensate reservoir's separators using Matlab software and other commercial simulators such as Aspen-Plus, Aspen-Hysys, and PVTi to do a comparison. This simulator is in good agreement with other 


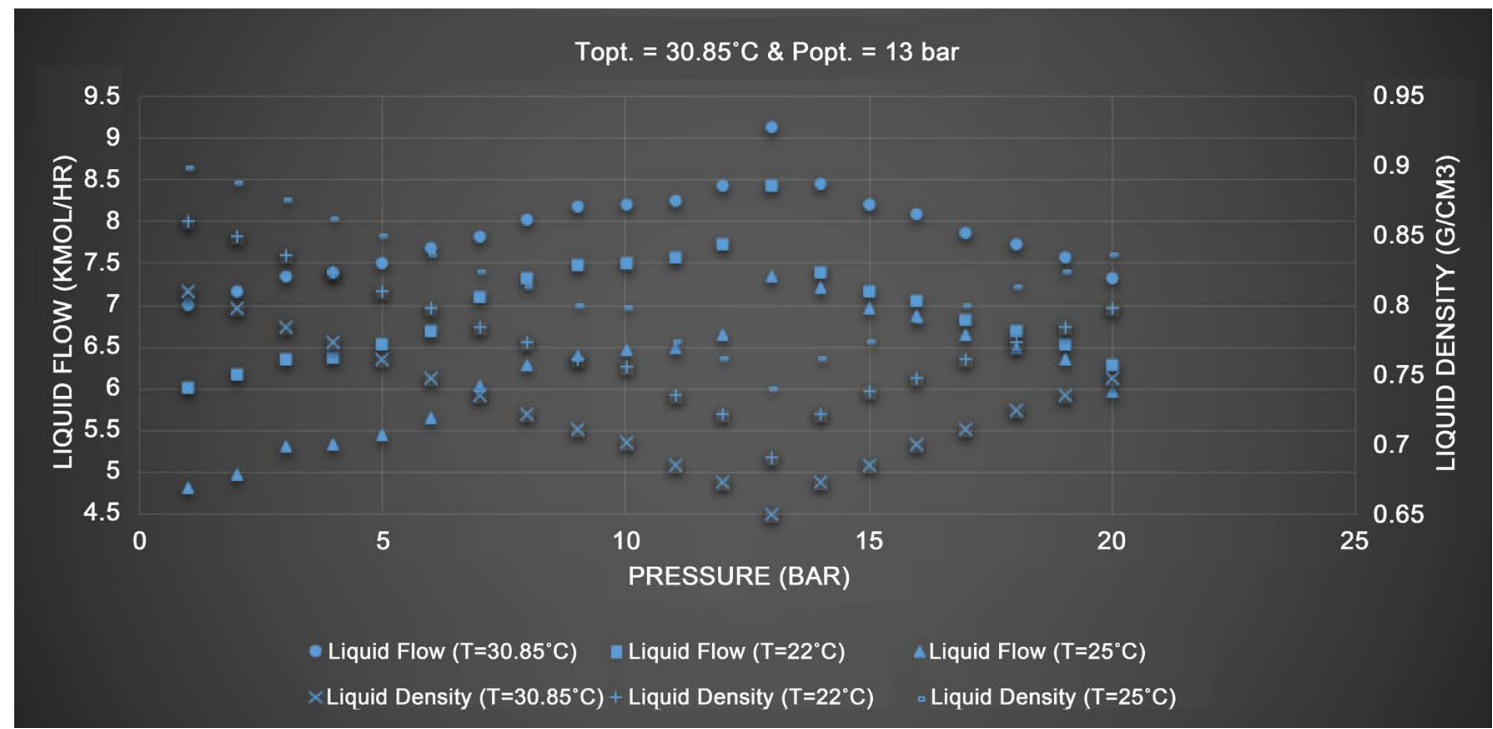

Figure 11. Optimum pressure and temperature for third separator in code analysis.

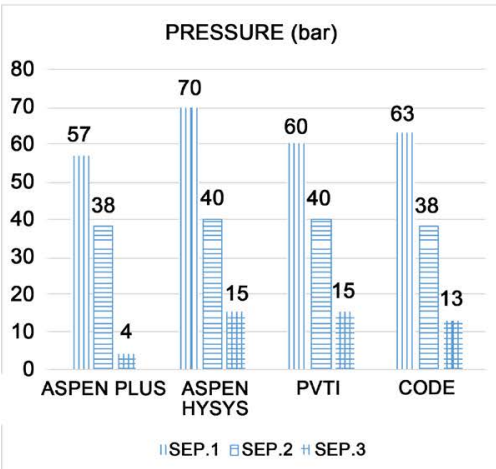

(a)



(b)

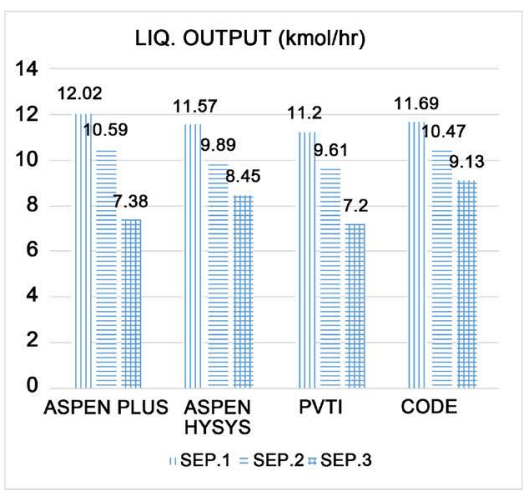

(c)

Figure 12. (a) Optimum pressure; (b) Optimum temperature; (c) Liquid output volume calculated from different simulators.

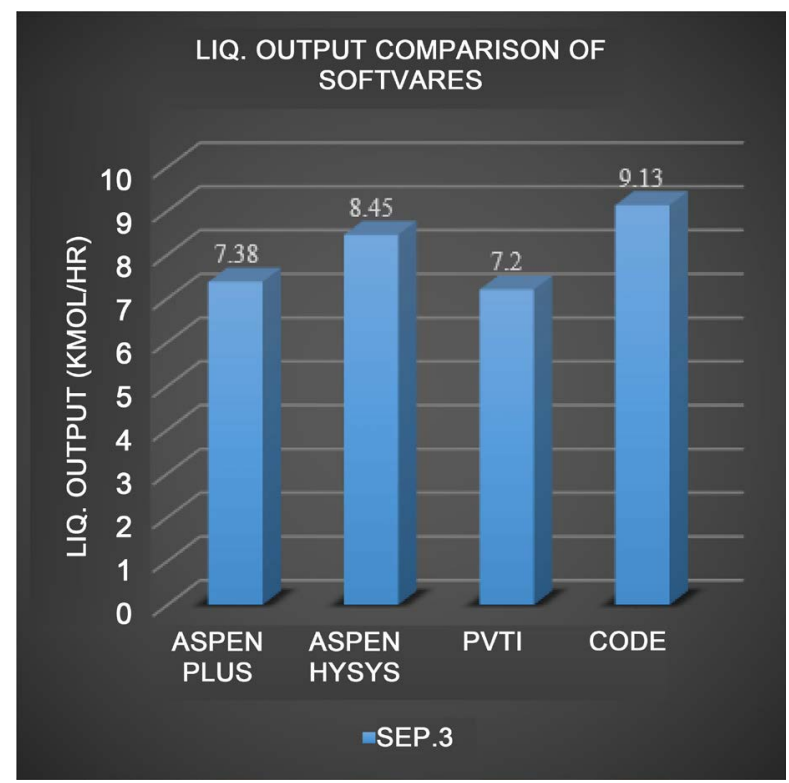

Figure 13. Comparison of liquid output calculated from third separator. 
simulators to predict the required parameters.

Also, this simulator is an easy-to-use simulator that the required parameters are directly obtained from it with the help of a simple algorithm.

Additionally, this simulator considers temperature variation with pressure variation simultaneously, and also this simulator is able to show optimum pressure and temperature between any ranges of pressures and temperatures that the user enters into this simulator. So, calculations and optimizations are done without any manual working. Finally, we can see the effect of various parameters on the optimum parameters in a so little runtime.

By considering the effect of both the pressure and the temperature in the optimum parameters (the stock tank liquid volume and the density), this simulator gives the highest amount of liquid volume into the stock tank in comparison to the other commercial simulators.

Also, by considering high amount of produced fluid in the wellhead, if the increased produced liquid volume which is predicted by the simulator is so little, the increased produced liquid volume which is practically predicted is so much in volume, because of the difference in the units. Therefore, it has very economical advantages.

Eventually, this simulator can be coupled with the other simulators to separator analysis with high accuracy.

\section{Acknowledgements}

The acknowledgments are for the Shiraz University for supporting this research.

\section{References}

[1] Ahmed, T. (2010) Reservoir Engineering Handbook. 4th Edition, Gulf Professional Publishing, Texas.

[2] Danesh, A. (1998) PVT and Phase Behavior of Petroleum Reservoir Fluids. Elsevier, Amsterdam.

[3] Firoozabadi, A. (1999) Thermodynamics of Hydrocarbon Reservoirs. McGraw-Hill, Pennsylvania Plaza, New York.

[4] Arnold, K. and Stewart, M. (2008) Surface Production Operations. 3rd Edition, Gulf Professional Publishing, Texas.

[5] Assael, M.J., Trusler, M. and Tsolakis, T.F. (1996) Thermo Physical Properties of Fluids: An Introduction to Their Prediction. Imperial College Press, London. https://doi.org/10.1142/p007

[6] Adjiman, C.S., Dallwig, S., Floudas, C.A. and Neumaier, A. (1998) A Global Optimization Method, Alfa BB, for General Twice-Differentiable Constrained NLPs Theoretical Advances. Computers \& Chemical Engineering, 22, 1137-1158. https://doi.org/10.1016/S0098-1354(98)00027-1

[7] Harding, S.T. and Floudas, C.A. (2000) Phase Stability with Cubic Equations of State: Global Optimization Approach. American Institute of Chemical Engineers Journal, 46, 1422-1440. https://doi.org/10.1002/aic.690460715

[8] Zhu, Y., Wen. H. and Xu, Z. (2000) Global Stability Analysis and Phase Equilibrium Calculations at High Pressures Using the Enhanced Simulated Annealing Algo- 
rithm. Chemical Engineering Science, 55, 3451-3459.

https://doi.org/10.1016/S0009-2509(00)00015-4

[9] Vázquez-Román, R., García-Sánchez, F., Salas-Padrón, A., Hernández-Garduza, O. and Eliosa-Jiménez, G. (2000) An Efficient Flash Procedure Using Cubic Equations of State. Chemical Engineering Journal, 84, 201-205. https://doi.org/10.1016/S1385-8947(00)00276-X

[10] Nichita, D.V., Gomez, S. and Luna, E. (2002) Multiphase Equilibria Calculation by Direct Minimization of Gibbs Free Energy with a Global Optimization Method. Computers \& Chemical Engineering, 26, 1703-1724. https://doi.org/10.1016/S0098-1354(02)00144-8

[11] Chaikunchuensakun, S., Stiel, L.I. and Baker, E.L. (2002) A Combined Algorithm for Stability and Phase Equilibrium by Gibbs Free Energy Minimization. Industrial \& Engineering Chemistry Research, 41, 4132-4140. https://doi.org/10.1021/ie011030t

[12] Zhou, H., Sun, W.M. and Xia, N. (2004) Application of CFD in the Modification of an Oil-Gas Separator Design. Journal of Hydrodynamic (Ser. A), 19, 926-929.

[13] Zhang, L.H., Xiao, H., Zhang, H.T., Xu, L.J. and Zhang, D. (2007) Optimal Design of a Novel Oil-Water Separator for Raw Oil Produced from ASP Flooding. Journal of Petroleum Science and Engineering, 59, 213-218. https://doi.org/10.1016/j.petrol.2007.04.002

[14] Bahadori, A., Vuthaluru, H.B. and Mokhatab, S. (2008) Optimizing Separator Pressures in the Multistage Crude Oil Production Unit. Asia-Pacific Journal of Chemical Engineering, 3, 380-386. https://doi.org/10.1002/apj.159

[15] Rossi, C.C., Cardozo-Filho, L. and Guirardello, R. (2009) Gibbs Free Energy Next Term Minimization for the Calculation of Chemical and Phase Equilibrium using Linear Programming. Fluid Phase Equilibria, 278, 117-128. https://doi.org/10.1016/j.fluid.2009.01.007

[16] Carroll, J. (2014) Natural Gas Hydrates: A Guide for Engineers. Gulf Professional Publishing, Houston.

[17] Ejraei Bakyani, A., Sahebi, H., Ghiasi, M.M., Mirjordavi, N., Esmaeilzadeh, F., Lee, M. and Bahadori, A. (2016) Prediction of $\mathrm{CO}_{2}$-Oil Molecular Diffusion using Adaptive Neuro-Fuzzy Inference System and Particle Swarm Optimization Technique. Fuel, 181, 178-187. https://doi.org/10.1016/j.fuel.2016.04.097

[18] Peng, D.Y. and Robinson, D.B. (1976) A New Two-Constant Equation of State. Industrial \& Engineering Chemistry Fundamentals, 15, 59-64. https://doi.org/10.1021/i160057a011

[19] Soave, G. (1972) Equilibrium Constants from a Modified Redlich-Kwong Equation of State. Chemical Engineering Science, 27, 1197-1203. https://doi.org/10.1016/0009-2509(72)80096-4

[20] Wilson, G. (1968) A Modified Redlich-Kwong EOS, Application to General Physical Data Calculations. American Institute of Chemical Engineers 65th National Meeting, Paper No. 15C. 


\section{Appendix (A)}

\section{Economic Analysis of the Developed Simulator}

Simulator's feed is calculated as $\mathrm{kmol} / \mathrm{hr}(100 \mathrm{kmol} / \mathrm{hr})$, but field's feed is calculated as bbl/day (5000 bbl/day for example). So, $100 \mathrm{kmol} / \mathrm{hr}$ is equivalent to $5000 \mathrm{bbl} /$ day. If stock tank liquid calculated from various simulators is different (CODE and ASPEN HYSYS) and this difference was $0.68 \mathrm{kmol} / \mathrm{hr}(9.13 \mathrm{kmol} / \mathrm{hr}$ $-8.45 \mathrm{kmol} / \mathrm{hr}$ ), so it is equivalent to a high amount of bbl liquid in several years by applying the appropriate conversion factor.

$$
\begin{aligned}
& 0.68 \mathrm{kmol} / \mathrm{hr} \times 147.625 \mathrm{kgr} / \mathrm{kmol} \times \frac{1}{0.762066} \mathrm{lit} / \mathrm{kgr} \\
& \times \frac{1}{160} \mathrm{bbl} / \mathrm{lit} \times 24 \mathrm{hr} / \text { day } \approx 20 \mathrm{bbl} / \text { day }
\end{aligned}
$$

For 5 years:

$$
5 \text { year } \times 365 \text { day } / \text { year } \times 20 \text { bbl/day } \approx 36500 \mathrm{bbl}
$$

Economical view:

$$
36500 \mathrm{bbl} \times 40 \$ / \mathrm{bbl} \approx 1460000 \$
$$




\section{Nomenclature}

\section{T Temperature}

P Pressure

Fraction $_{\mathrm{VAP}}$. Vapor fraction in input flow to separators

Fraction $_{\text {LIQ. }}$ Liquid fraction in input flow to separators



E Enthalpy

S Entropy

$\rho \quad$ Average density

$\rho_{\mathrm{L}} \quad$ Liquid density

$\rho_{\mathrm{V}} \quad$ Vapor density

$\mathrm{MW}_{\mathrm{AV}} \quad$ Average molecular weight

$\mathrm{MW}_{\mathrm{AV} . \mathrm{L}} \quad$ Liquid average molecular weight

$\mathrm{MW}_{\mathrm{AV} . \mathrm{V}} \quad$ Vapor average molecular weight

$\mathrm{V}_{\mathrm{L}} \quad$ Liquid volume

$\mathrm{V}_{\mathrm{V}} \quad$ Vapor volume

Q Heat rate

GOR Gas oil ratio

$\mathrm{B}_{\mathrm{O}} \quad$ Oil formation volume factor

$\mathrm{z}_{\mathrm{i}} \quad$ Mole percent of $\mathrm{i}$-component in two phase flow

$\mathrm{x}_{\mathrm{i}} \quad$ Mole percent of $\mathrm{i}$-component in liquid phase

$y_{i} \quad$ Mole percent of $\mathrm{i}$-component in vapor phase 